Published in final edited form as:

Clin Rheumatol. 2018 February ; 37(2): 467-474. doi:10.1007/s10067-017-3860-x.

\title{
Discordance of the Framingham cardiovascular risk score and the 2013 American College of Cardiology/American Heart Association risk score in systemic lupus erythematosus and rheumatoid arthritis
}

\author{
Kashif Jafri, MD, \\ Department of Medicine, Division of Rheumatology, University of California, San Francisco, 513 \\ Parnassus Avenue Box 0500, Medical Sciences Room S847, San Francisco, CA 94143

\begin{abstract}
Alexis Ogdie, MD, MSCE,
Department of Medicine, Division of Rheumatology, University of Pennsylvania, White Building, Room 5024, 3400 Spruce Street, Philadelphia, PA 19104

Atif Qasim, MD, MSCE,

Department of Medicine, Division of Cardiology, University of California, San Francisco, 505

Parnassus Avenue, M1177B, San Francisco, CA 94143
\end{abstract}

\section{Sarah L. Patterson, MD,}

Department of Medicine, Division of Rheumatology, University of California, San Francisco, 513

Parnassus Avenue Box 0500, Medical Sciences Room S847, San Francisco, CA 94143

\author{
Milena Gianfrancesco, PhD, MPH, \\ Department of Medicine, Division of Rheumatology, University of California, San Francisco, UCSF \\ Box 0500, 513 Parnassus Avenue, San Francisco, CA 94143

\section{Zara Izadi, PharmD, \\ Department of Epidemiology and Biostatistics, University of California, San Francisco, $55016^{\text {th }}$ \\ Street, San Francisco, CA 94158}

Patricia Katz, PhD,

Department of Medicine, Division of Rheumatology, University of California, San Francisco, UCSF Box 0920, 3333 California Street, San Francisco, CA 94143-0920

Jinoos Yazdany, MD, MPH, and

\footnotetext{
Corresponding Author: Kashif Jafri, MD, Department of Medicine, Division of Rheumatology, University of California, San Francisco, 513 Parnassus Avenue Box 0500, Medical Sciences Room S847, San Francisco, CA 94143, Telephone number: 415-750-2104, Fax number: 415-750-6920, kashif.jafri@ucsf.edu.

Transparency declaration: The authors affirm that the manuscript is an honest, accurate, and transparent account of the study being reported.

Contributions:

Study conception and design Jafri, Ogdie, Qasim, Patterson, Gianfrancesco, Izadi, Katz, Yazdany, Schmajuk Acquisition of data Jafri, Katz, Yazdany, Schmajuk

Analysis and interpretation of data - Jafri, Ogdie, Qasim, Patterson, Gianfrancesco, Izadi, Katz, Yazdany, Schmajuk

Competing interests: Kashif Jafri, Alexis Ogdie, Sarah L. Patterson, Milena Gianfrancesco, Zara Izadi, and Patricia Katz have no financial disclosures. Atif Qasim receives research support from Medtronic and Abbott Vascular. Jinoos Yazdany and Gabriela Schmajuk have received an investigator-initiated research grant from Pfizer.
} 
Department of Medicine, Division of Rheumatology, University of California, San Francisco, 533

Parnassus Avenue, San Francisco, CA 94143

\section{Gabriela Schmajuk, MD, MSc}

Department of Medicine, Division of Rheumatology, University of California, San Francisco, San

Francisco VA Medical Center, 4150 Clement St, Mailstop 111R, San Francisco, CA 94121

\section{Abstract}

Objectives-Despite increasing use of the 2013 American College of Cardiology/American Heart Association (ACC/AHA) cardiovascular (CV) risk score in clinical practice, few studies have compared this score to the Framingham risk score among rheumatologic patients. We calculated Framingham and 2013 ACC/AHA risk scores in subjects with systemic lupus erythematosus (SLE) and rheumatoid arthritis (RA), and assessed demographic, CV, and rheumatologic characteristics associated with discordant scores (high risk ACC/AHA scores but low risk Framingham scores).

Methods-SLE and RA subjects drawn from 2 population-based cohort studies were assessed during in-person study visits. We used chi-squared tests and t-tests to examine the association of discordant $\mathrm{CV}$ risk scores with baseline characteristics.

Results-11 (7.0\%) of 157 SLE subjects and 11 (11.5\%) of 96 RA subjects had discordant CV risk scores with high ACC/AHA scores and low Framingham scores. These findings did not significantly change when a 1.5 multiplier was applied to the Framingham score. Rheumatologic disease duration, high-sensitivity CRP levels, African-American race, diabetes, current use of antihypertensive medication, higher age, and higher systolic blood pressure were each significantly associated with discordant risk scores.

Conclusions-Approximately $10 \%$ of SLE and RA subjects had discordant 10-year CV risk scores. Our findings suggest that the use of the 2013 ACC/AHA risk score could result in changes to lipid-lowering therapy recommendations in a significant number of rheumatologic patients. Prospective studies are needed to compare which score better predicts CV events in rheumatologic patients, especially those with risk factors associated with discordant risk scores.

\section{Introduction}

Patients with systemic lupus erythematosus (SLE) or rheumatoid arthritis (RA) have an increased risk for cardiovascular disease compared to the general population, and cardiovascular events are a major cause of morbidity and mortality in these populations [1,2]. The increased cardiovascular risk in patients with SLE and RA appears to be explained by multiple factors including a high prevalence of traditional cardiovascular risk factors (such as hypertension and hyperlipidemia), systemic inflammation related to the underlying rheumatologic disease, and chronic corticosteroid use [2,3]. Previous studies have demonstrated that traditional cardiovascular risk factors are highly prevalent, underdiagnosed, and poorly controlled in this patient population $[4,5]$.

The Framingham cardiovascular risk score (which includes variables for age, sex, total cholesterol, HDL cholesterol, systolic blood pressure, smoking status, and use of anti- 
hypertensive medication) has been used to perform cardiovascular risk stratification in the general population, but it underestimates cardiovascular risk in young patients, women, and patients with inflammatory diseases such as SLE and RA [6]. Multipliers to traditional 10year cardiovascular risk scores have therefore been proposed to more accurately characterize cardiovascular risk in patients with certain rheumatologic conditions. For example, the European Union League Against Rheumatism (EULAR) has published recommendations for RA that advise physicians to calculate a 10-year coronary heart disease risk (such as a 10year Framingham risk score) for each patient, to multiply this risk by a factor of 1.5, and to subsequently use the adjusted risk score to manage cardiovascular risk [7]. In 2013, the American College of Cardiology/American Heart Association (ACC/AHA) developed a risk assessment tool for the general population. This tool incorporates the additional variables of race and diabetes, but the presence of a rheumatologic disease does not influence the 2013 ACC/AHA cardiovascular risk calculation [8,9].

From the public health perspective, cardiovascular risk stratification generally occurs in the primary care setting without accounting for specific rheumatologic factors such as disease activity or disease duration. Despite increasing use of the 2013 ACC/AHA cardiovascular risk score in clinical practice in the United States, few studies have compared this score to the Framingham risk score in patients with rheumatologic conditions $[9,6]$. We aimed to conduct a highly pragmatic study to understand how the commonly used cardiovascular risk scores in primary care in the United States differentially stratify patients with SLE and RA, which is an important first step in analyzing the clinical utility of the new 2013 ACC/AHA risk score in patients with rheumatic diseases. The objectives of our cross-sectional study were: 1) to determine the proportion of discordant 10-year Framingham cardiovascular risk scores and 2013 ACC/AHA cardiovascular risk scores in subjects with SLE and RA, 2) to determine the proportion of discordant 10-year modified Framingham cardiovascular risk scores (with the EULAR-proposed1.5 multiplier) and 2013 ACC/AHA cardiovascular risk scores in these subjects, and 3) to assess demographic, cardiovascular, and rheumatologic clinical characteristics associated with discordant cardiovascular risk scores.

\section{Methods}

\section{Data Source and Study Population}

In this cross-sectional study, the subjects in the SLE and RA groups had participated in the Arthritis, Body Composition, and Disability (ABCD) Study. Patients included in the ABCD Study were drawn from 2 longstanding cohort studies (the University of California, San Francisco, Lupus Outcomes Study and RA Panel Study). The subjects were initially recruited from both clinical and community-based sources in Northern California and had in-person research visits; additional details regarding these data sources have been reported previously $[10,11]$. All subjects had diagnoses of SLE or RA that were based on the American College of Rheumatology criteria and verified by medical record review. Exclusion criteria for the ABCD Study included non-English-speaking status, current daily oral prednisone of $50 \mathrm{mg}$ or greater, current pregnancy, uncorrected vision problems that would interfere with reading ability, and joint replacement within 1 year. Additional 
exclusion criteria for the present analysis were the absence of data required to calculate the Framingham or 2013 ACC/AHA risk scores $(\mathrm{n}=14)$.

Although the Framingham risk score is traditionally applied to patients without diabetes, we did not exclude subjects with diabetes in this study in order to perform an analysis of whether the presence of diabetes was associated with discordance of the Framingham risk score and the 2013 ACC/AHA risk score. A sensitivity analysis that excluded subjects with diabetes was performed to allow for comparison of the results. In addition, the 2013 ACC/AHA pooled cohort equations are intended to be applied in patients with age 40-75 years, total cholesterol 130-320 mg/dL, HDL cholesterol 20-320 mg/dL, LDL cholesterol < $190 \mathrm{mg} / \mathrm{dL}$, and systolic blood pressure 90-200 $\mathrm{mmHg}$, but the values of certain variables required for calculation of the ACC/AHA risk score did not fall into this range in 59 subjects, most commonly because of age $<40(\mathrm{n}=41)$ and total cholesterol $<130 \mathrm{mg} / \mathrm{dL}$ $(n=15)[8,9]$. The values of these variables were imputed to the nearest value in the range required to calculate the ACC/AHA risk score, which is reflective of common clinical practice.

\section{Variables and Measurements}

Demographic characteristics, smoking status, diabetes status, rheumatologic disease duration, and current medication use data were obtained from subjects by self-report at an in-person research visit, and rheumatologic disease activity was assessed using either the Systemic Lupus Activity Questionnaire (SLAQ) or the Rheumatoid Arthritis Disease Activity Index (RADAI), which are validated self-reported measures of disease activity $[12,13]$. Blood pressures and weights were measured by registered nurses. Laboratory studies including high-sensitivity CRP (hsCRP), total cholesterol, LDL cholesterol, HDL cholesterol, and triglycerides were collected in non-fasting conditions; robust evidence supports the use of non-fasting blood draws for routine lipid testing [14]. The 10-year Framingham cardiovascular risk score (which includes age, sex, total cholesterol, HDL cholesterol, systolic blood pressure, smoking status, and current use of anti-hypertensive medication) and 2013 ACC/AHA cardiovascular risk score (which includes race and diabetes status in addition to the aforementioned Framingham variables) were subsequently calculated for each subject. A modified 10-year Framingham cardiovascular risk score with a 1.5 multiplier was also calculated for the purpose of comparison given the EULAR recommendations noted above.

As per Adult Treatment Panel-III (ATP-III) recommendations and previous related studies, a subject with a 10-year Framingham risk score (or modified Framingham risk score with a 1.5 multiplier) $\geq 10 \%$ was defined as high-risk by that score, whereas a subject with a 10 year Framingham risk score (or modified Framingham risk score with a 1.5 multiplier) < $10 \%$ was defined as low-risk[15,6]. A subject with a 10-year 2013 ACC/AHA risk score $\geq$ 7.5\% was defined as high-risk by that score, whereas a subject with a 10-year 2013 ACC/AHA risk score $<7.5 \%$ was defined as low-risk. The $7.5 \%$ threshold identifies patients who would benefit from lipid-lowering therapy according to these recent ACC/AHA guidelines [9]. A subject with a discordant cardiovascular risk score was defined as one who had a Framingham risk score (or modified Framingham risk score with a 1.5 multiplier) that 
characterized him/her as low-risk and an ACC/AHA risk score that characterized him/her as high risk, or vice versa.

\section{Statistical analysis}

Associations of demographic, cardiovascular, and rheumatologic characteristics with discordant cardiovascular risk scores were analyzed using chi-squared tests for categorical variables and using independent t-tests for continuous variables. All statistical analyses were performed using Stata software, version 13.0.

\section{Results}

We included 157 subjects with SLE and 96 subjects with RA. The mean age of the SLE subjects was 50.1 years, and the mean age of the RA subjects was 58.7 years (Table 1 ). In the SLE group, $93.0 \%$ of the subjects were female and $12.7 \%$ were African-American. In the RA group, $83.3 \%$ of the subjects were female and $2.1 \%$ were African-American. On average, the SLE and RA subjects were overweight with a mean body mass index (BMI) of $27.4 \mathrm{~kg} / \mathrm{m}^{2}$ and $26.3 \mathrm{~kg} / \mathrm{m}^{2}$, respectively. The mean rheumatologic disease duration for both groups was over 15 years. Nearly $50 \%$ of SLE subjects were using prednisone therapy with a mean daily prednisone dose of $8.1 \mathrm{mg}$ among prednisone users; $29.0 \%$ of RA subjects were taking prednisone with a mean daily prednisone dose of $6.1 \mathrm{mg}$ among prednisone users. $20.4 \%$ of SLE subjects were receiving statin therapy; $76.0 \%$ of RA subjects had missing data on statin use, and of the 23 RA subjects with available data on statin use, 16 $(69.6 \%)$ were using statins.

The majority of subjects (over 75\%) in both groups had concordant low cardiovascular risk scores, with a low Framingham risk score and a low ACC/AHA risk score (Table 2). 7.0\% $(n=11)$ of SLE subjects and $11.5 \%(n=11)$ of RA subjects had discordant cardiovascular risk scores with low Framingham risk scores but high ACC/AHA risk scores. None of the subjects had a high Framingham risk score and low ACC/AHA risk score. When the 1.5 multiplier was applied to the Framingham risk score, 3 additional subjects with RA were identified as high risk and the number of subjects with discordant risk scores increased by only 1 (from $8.7 \%$ to $9.1 \%$ ).

We compared the 22 subjects who had discordant Framingham and ACC/AHA risk scores (FRS-low/ACC-high)with the 231 subjects who had concordant risk scores. Among the 22FRS-low/ACC-high subjects, the mean hsCRP level was $7.3 \mathrm{mg} / \mathrm{L}$ and the mean rheumatologic disease duration was 23.5 years compared to $3.6 \mathrm{mg} / \mathrm{L}$ and 17.4 years, respectively, in subjects with concordant cardiovascular risk scores ( $\mathrm{p} \unlhd \mathbf{\Delta} .01$ ). AfricanAmerican race, diabetes, current use of anti-hypertensive medication, higher age, and higher systolic blood pressure were also associated with discordant FRS-low/ACC-high scores (p $₫$.05). In a sensitivity analysis excluding 14 subjects with diabetes, only age, systolic blood pressure, and rheumatologic disease duration were significantly associated with discordant risk scores ( $\mathrm{p} \unlhd .05$ ).

When the 59 subjects who required imputation of variables for calculation of the ACC/AHA risk score were excluded from the analysis, the percentage of FRS-low/ACC-high subjects 
remained similar with $8.8 \%$ of SLE subjects and $9.9 \%$ of RA subjects having this discordant risk score. When high risk by the 10-year 2013 ACC/AHA risk score was redefined as $\geq$ $10 \%$, the number of FRS-low/ACC-high SLE subjects decreased from 11 to 10, and the number of FRS-low/ACC-high RA subjects decreased from 11 to 8 .

\section{Discussion}

Approximately $10 \%$ of both SLE and RA subjects in this study had discordant 10-year cardiovascular risk scores with low Framingham risk scores and high 2013 ACC/AHA risk scores. None of the subjects had a high Framingham risk score and low ACC/AHA risk score, indicating that the 2013 ACC/AHA risk score may classify more SLE and RA patients as high risk. We found no significant change in the proportion of discordant subjects after applying a 1.5 multiplier to the Framingham risk score. Interestingly, hsCRP levels and rheumatologic disease duration were significantly associated with discordant FRS-low/ACChigh risk scores, suggesting that the ACC/AHA risk score may better capture exposure to systemic inflammation. Multiple other demographic and cardiovascular clinical characteristics that contributed to one or both of the cardiovascular risk scores under study were also significantly associated with discordant FRS-low/ACC-high risk scores, including age, African-American race, systolic blood pressure, diabetes, and current use of antihypertensive medication. SLE and RA patients with these characteristics may therefore be more likely to receive recommendations for lipid-lowering therapy if the ACC/AHA risk score is used.

A recent study using the National Health and Nutrition Examination Surveys of 2005 to 2010 estimated that the number of U.S. adults eligible for statin therapy would increase by 12.8 million (from $37.5 \%$ to $48.6 \%$ ) based on the new ACC/AHA guidelines compared to the ATP-III guidelines, but few studies have compared the 2013 ACC/AHA risk score and the Framingham risk score in patients with rheumatologic diseases [16]. A study by Kawai et al. addressed the ability of the 2013 ACC/AHA risk score to identify RA patients with high coronary artery calcification scores and demonstrated that $16 \%$ of patients categorized as low risk by the Framingham risk score were reclassified as high risk by the ACC/AHA risk score [6]. Our study had similar results with $11.5 \%$ of subjects in the RA cohort who were low risk by the Framingham risk score, yet recategorized as high risk by the ACC/AHA risk score. Overall, our findings suggest that the use of the 2013 ACC/AHA risk score would result in changes in management recommendations for initiation of lipidlowering therapy and for more aggressive lifestyle modification in a significant number of rheumatologic patients. Consistent with that notion, a previous French multicenter study of 677 statin-naïve RA patients demonstrated that statins would be recommended in $78.5 \%$ of men and $38.8 \%$ of women according to the 2013 ACC/AHA guidelines compared to only $53.1 \%$ of men and $15.6 \%$ of women according to the ATP-III guidelines [17]. The results of our study are difficult to directly compare to that study given that our study addressed discordance in risk categorization rather than discordance in recommendations for statin therapy in statin-naïve patients, but the higher rate of discordance in that study may be explained by application of the EULAR 1.5 multiplier to both risk scores in nearly $60 \%$ of subjects (depending on specific RA characteristics). 
The ability of the 2013 ACC/AHA risk score to accurately identify rheumatologic patients with high cardiovascular risk is still unclear. In a recent study of 216 RA patients by Ozen et al., the 2013 ACC/AHA risk score failed to identify about $55 \%$ of the subjects with increased carotid intima-media thickness and/or carotid plaques, and Kawai et al. reported that the 2013 ACC/AHA risk score assigned almost $60 \%$ of patients with high coronary artery calcium into a low risk category $[18,6]$. Future studies should investigate whether the risk categorization of SLE and RA patients by the ACC/AHA risk score correlates not only with surrogate markers of cardiovascular disease, but also with long-term cardiovascular events. Importantly, the values of certain variables required for calculation of the ACC/AHA risk score did not fall into the intended range in 59 subjects and necessitated imputation of these variables. The most common reason for this imputation was age $<40$, underscoring the importance of developing cardiovascular risk assessment tools that are applicable and validated in young patients with rheumatic diseases.

The present study has several strengths. To our knowledge, this is the first study that has addressed the discordance of the Framingham risk score and the 2013 ACC/AHA risk score in over 250 subjects with RA or SLE. RA-specific cardiovascular risk scores have been proposed, but our study provides a practical comparison of the Framingham risk score (with and without a 1.5 multiplier) and the 2013 ACC/AHA risk score, which are commonly used in clinical practice [19]. The results have important clinical implications because indications for lipid-lowering therapy are based on these cardiovascular risk scores. Nevertheless, this study has some limitations. The study population was recruited in California and was predominantly Caucasian, which may limit the generalizability of the results to other populations. Disease activity scores that include objective laboratory data and physician assessments were not available, though we did assess validated self-reported disease activity measures. Longitudinal clinical data such as cumulative steroid use, NSAID use, and use of other immunosuppressive therapy were not available. Although data on use of statins were also unavailable for $76 \%$ of the RA subjects, statin use would not invalidate the clinical utility of cardiovascular risk assessment tools. However, statins have pleiotropic antiinflammatory effects and may affect disease activity in SLE and/or RA, though this is controversial and quantification of a potential effect on disease activity is difficult given the number of confounding factors [20,21]. Other cardiovascular risk scores, such as the QRESEARCH Cardiovascular Risk Algorithm 2 (QRISK 2), were not assessed in this study given the absence of necessary data and our objective to determine differences in cardiovascular risk scores that are used in the "real-world" primary care setting; a comparison of other cardiovascular risk scores is an exciting area for future research.

In summary, a small but significant fraction of SLE and RA subjects had discordant cardiovascular risk scores with low Framingham risk scores and high ACC/AHA risk scores, even when a 1.5 multiplier was applied to the Framingham risk score. Prospective studies are needed to address the ability of different cardiovascular risk assessment tools to predict cardiovascular events in rheumatologic patients. These studies have the potential to provide a more evidence-based approach to cardiovascular risk stratification and reduce cardiovascular morbidity and mortality in patients with rheumatologic diseases. 


\section{Acknowledgments}

Funding: NIH T32 5T32AR007304-38 (KJ); NIH/NIAMS P60 AR053308 (PK); AHRQ R01 HS024412 (JY); NIH/NIAMS P30 AR070155 (JY); NIH/NIAMS K23 AR063770 (GS)

We thank C. Michael Stein, MB, ChB, Dan May Professor of Medicine and Pharmacology at Vanderbilt University, for reviewing the manuscript and providing helpful comments.

\section{References}

1. Avina-Zubieta JA, Thomas J, Sadatsafavi M, Lehman AJ, Lacaille D. Risk of incident cardiovascular events in patients with rheumatoid arthritis: a meta-analysis of observational studies. Annals of the rheumatic diseases. 2012; 71(9):1524-1529. DOI: 10.1136/ annrheumdis-2011-200726 [PubMed: 22425941]

2. Magder LS, Petri M. Incidence of and risk factors for adverse cardiovascular events among patients with systemic lupus erythematosus. Am J Epidemiol. 2012; 176(8):708-719. DOI: 10.1093/aje/ kws130 [PubMed: 23024137]

3. Croca SC, Rahman A. Imaging assessment of cardiovascular disease in systemic lupus erythematosus. Clin Dev Immunol. 2012; 2012:694143.doi: 10.1155/2012/694143 [PubMed: 22110536]

4. Primdahl J, Clausen J, Horslev-Petersen K. Results from systematic screening for cardiovascular risk in outpatients with rheumatoid arthritis in accordance with the EULAR recommendations. Annals of the rheumatic diseases. 2013; 72(11):1771-1776. DOI: 10.1136/ annrheumdis-2013-203682 [PubMed: 23852694]

5. Bartels CM, Johnson H, Voelker K, Thorpe C, McBride P, Jacobs EA, Pandhi N, Smith M. Impact of rheumatoid arthritis on receiving a diagnosis of hypertension among patients with regular primary care. Arthritis care \& research. 2014; 66(9):1281-1288. DOI: 10.1002/acr.22302 [PubMed: 24585741]

6. Kawai VK, Chung CP, Solus JF, Oeser A, Raggi P, Stein CM. The ability of the 2013 American College of Cardiology/American Heart Association cardiovascular risk score to identify rheumatoid arthritis patients with high coronary artery calcification scores. Arthritis Rheumatol. 2015; 67(2): 381-385. DOI: 10.1002/art.38944 [PubMed: 25371313]

7. Agca R, Heslinga SC, Rollefstad S, Heslinga M, McInnes IB, Peters MJ, Kvien TK, Dougados M, Radner H, Atzeni F, Primdahl J, Sodergren A, Wallberg Jonsson S, van Rompay J, Zabalan C, Pedersen TR, Jacobsson L, de Vlam K, Gonzalez-Gay MA, Semb AG, Kitas GD, Smulders YM, Szekanecz Z, Sattar N, Symmons DP, Nurmohamed MT. EULAR recommendations for cardiovascular disease risk management in patients with rheumatoid arthritis and other forms of inflammatory joint disorders: 2015/2016 update. Annals of the rheumatic diseases. 2017; 76(1):1728. DOI: 10.1136/annrheumdis-2016-209775 [PubMed: 27697765]

8. Goff DC Jr, Lloyd-Jones DM, Bennett G, Coady S, D’ Agostino RB Sr, Gibbons R, Greenland P, Lackland DT, Levy D, O’Donnell CJ, Robinson JG, Schwartz JS, Shero ST, Smith SC Jr, Sorlie P, Stone NJ, Wilson PW. American College of Cardiology/American Heart Association Task Force on Practice G. 2013 ACC/AHA guideline on the assessment of cardiovascular risk: a report of the American College of Cardiology/American Heart Association Task Force on Practice Guidelines. Journal of the American College of Cardiology. 2014; 63(25 PtB):2935-2959. DOI: 10.1016/j.jacc. 2013.11.005 [PubMed: 24239921]

9. Stone NJ, Robinson JG, Lichtenstein AH, Bairey Merz CN, Blum CB, Eckel RH, Goldberg AC, Gordon D, Levy D, Lloyd-Jones DM, McBride P, Schwartz JS, Shero ST, Smith SC Jr, Watson K, Wilson PW. American College of Cardiology/American Heart Association Task Force on Practice G. 2013 ACC/AHA guideline on the treatment of blood cholesterol to reduce atherosclerotic cardiovascular risk in adults: a report of the American College of Cardiology/American Heart Association Task Force on Practice Guidelines. Journal of the American College of Cardiology. 2014; 63(25 Pt B):2889-2934. DOI: 10.1016/j.jacc.2013.11.002 [PubMed: 24239923]

10. Katz P, Julian L, Tonner MC, Yazdany J, Trupin L, Yelin E, Criswell LA. Physical activity, obesity, and cognitive impairment among women with systemic lupus erythematosus. Arthritis care \& research. 2012; 64(4):502-510. DOI: 10.1002/acr.21587 [PubMed: 22337564] 
11. Katz PP, Yazdany J, Trupin L, Schmajuk G, Margaretten M, Barton J, Criswell LA, Yelin EH. Sex differences in assessment of obesity in rheumatoid arthritis. Arthritis care \& research. 2013; 65(1): 62-70. DOI: 10.1002/acr.21810 [PubMed: 22833513]

12. Yazdany J, Yelin EH, Panopalis P, Trupin L, Julian L, Katz PP. Validation of the systemic lupus erythematosus activity questionnaire in a large observational cohort. Arthritis and rheumatism. 2008; 59(1):136-143. DOI: 10.1002/art.23238 [PubMed: 18163398]

13. Fransen J, Langenegger T, Michel BA, Stucki G. Feasibility and validity of the RADAI, a selfadministered rheumatoid arthritis disease activity index. Rheumatology. 2000; 39(3):321-327. [PubMed: 10788543]

14. Mora S. Nonfasting for Routine Lipid Testing: From Evidence to Action. JAMA Intern Med. 2016; 176(7):1005-1006. DOI: 10.1001/jamainternmed.2016.1979 [PubMed: 27119719]

15. National Cholesterol Education Program Expert Panel on Detection E, Treatment of High Blood Cholesterol in A. Third Report of the National Cholesterol Education Program (NCEP) Expert Panel on Detection, Evaluation, and Treatment of High Blood Cholesterol in Adults (Adult Treatment Panel III) final report. Circulation. 2002; 106(25):3143-3421. [PubMed: 12485966]

16. Pencina MJ, Navar-Boggan AM, D’Agostino RB Sr, Williams K, Neely B, Sniderman AD, Peterson ED. Application of new cholesterol guidelines to a population-based sample. N Engl J Med. 2014; 370(15):1422-1431. DOI: 10.1056/NEJMoa1315665 [PubMed: 24645848]

17. Tournadre A, Tatar Z, Pereira B, Chevreau M, Gossec L, Gaudin P, Soubrier M, Dougados M. Application of the European Society of Cardiology, Adult Treatment Panel III and American College of Cardiology/American Heart Association guidelines for cardiovascular risk management in a French cohort of rheumatoid arthritis. Int J Cardiol. 2015; 183:149-154. DOI: 10.1016/ j.ijcard.2015.01.069 [PubMed: 25666124]

18. Ozen G, Sunbul M, Atagunduz P, Direskeneli H, Tigen K, Inane N. The 2013 ACC/AHA 10-year atherosclerotic cardiovascular disease risk index is better than SCORE and Q Risk II in rheumatoid arthritis: is it enough? Rheumatology. 2016; 55(3):513-522. DOI: 10.1093/rheumatology/kev363 [PubMed: 26472565]

19. Solomon DH, Greenberg J, Curtis JR, Liu M, Farkouh ME, Tsao P, Kremer JM, Etzel CJ. Derivation and internal validation of an expanded cardiovascular risk prediction score for rheumatoid arthritis: a Consortium of Rheumatology Researchers of North America Registry Study. Arthritis Rheumatol. 2015; 67(8):1995-2003. DOI: 10.1002/art.39195 [PubMed: 25989470]

20. Xing B, Yin YF, Zhao LD, Wang L, Zheng WJ, Chen H, Wu QJ, Tang FL, Zhang FC, Shan G, Zhang X. Effect of 3-hydroxy-3-methylglutaryl-coenzyme a reductase inhibitor on disease activity in patients with rheumatoid arthritis: a meta-analysis. Medicine (Baltimore). 2015; 94(8):e572.doi: 10.1097/MD.0000000000000572 [PubMed: 25715256]

21. Sahebkar A, Rathouska J, Derosa G, Maffioli P, Nachtigal P. Statin impact on disease activity and C-reactive protein concentrations in systemic lupus erythematosus patients: A systematic review and meta-analysis of controlled trials. Autoimmun Rev. 2016; 15(4):344-353. DOI: 10.1016/ j.autrev.2015.12.007 [PubMed: 26747436] 
Table 1

Demographic and Clinical Characteristics of SLE and RA Cohorts

\begin{tabular}{|c|c|c|c|}
\hline & & SLE (N=157) & RA $(\mathbf{N}=96)$ \\
\hline \multicolumn{4}{|l|}{$\underline{\text { Demographic Characteristics }}$} \\
\hline Age (years) & Mean \pm SD & $50.1 \pm 8.9$ & $58.7 \pm 9.1$ \\
\hline Female & $\mathrm{N}(\%)$ & $146(93.0 \%)$ & $80(83.3 \%)$ \\
\hline Caucasian race & $\mathrm{N}(\%)$ & $101(64.3 \%)$ & $81(84.4 \%)$ \\
\hline African-American race & $\mathrm{N}(\%)$ & $20(12.7 \%)$ & $2(2.1 \%)$ \\
\hline Asian race & $\mathrm{N}(\%)$ & $24(15.3 \%)$ & $6(6.3 \%)$ \\
\hline Hispanic race & $\mathrm{N}(\%)$ & $30(19.1 \%)$ & $12(12.5 \%)$ \\
\hline \multicolumn{4}{|l|}{ Cardiovascular Clinical Characteristics } \\
\hline Body mass index $\left(\mathrm{kg} / \mathrm{m}^{2}\right)$ & Mean \pm SD & $27.4 \pm 6.9$ & $26.3 \pm 5.2$ \\
\hline Systolic blood pressure $(\mathrm{mm} \mathrm{Hg})$ & Mean \pm SD & $121 \pm 20$ & $125 \pm 18$ \\
\hline Diastolic blood pressure ( $\mathrm{mm} \mathrm{Hg}$ ) & Mean \pm SD & $70 \pm 14$ & $71 \pm 10$ \\
\hline Total cholesterol (mg/dL) & Mean \pm SD & $179 \pm 35$ & $193 \pm 40$ \\
\hline LDL cholesterol (mg/dL) & Mean \pm SD & $92 \pm 31$ & $102 \pm 34$ \\
\hline HDL cholesterol (mg/dL) & Mean \pm SD & $61 \pm 18$ & $67 \pm 20$ \\
\hline Triglycerides (mg/dL) & Mean \pm SD & $127 \pm 72$ & $112 \pm 50$ \\
\hline Current smoker & $\mathrm{N}(\%)$ & $10(6.4 \%)$ & $5(5.2 \%)$ \\
\hline Diabetes & $\mathrm{N}(\%)$ & $11(7.0 \%)$ & $3(3.1 \%)$ \\
\hline Current use of anti-hypertensive medication & $\mathrm{N}(\%)$ & $66(42.0 \%)$ & $34(35.4 \%)$ \\
\hline Current use of statin & $\mathrm{N}(\%)$ & $32(20.4 \%)$ & $*$ \\
\hline \multicolumn{4}{|l|}{ Rheumatologic Clinical Characteristics } \\
\hline hsCRP (mg/L) & Mean \pm SD & $3.9 \pm 6.8$ & $4.0 \pm 6.0$ \\
\hline Current use of prednisone therapy ${ }^{* *}$ & $\mathrm{~N}(\%)$ & $70(45.5 \%)$ & $27(29.0 \%)$ \\
\hline Current daily prednisone dose among prednisone users (mg) & Mean \pm SD & $8.1 \pm 4.6$ & $6.1 \pm 3.7$ \\
\hline Disease duration (years) & Mean \pm SD & $15.8 \pm 9.3$ & $21.4 \pm 11.6$ \\
\hline SLAQ score & Mean \pm SD & $12.6 \pm 7.5$ & Not applicable \\
\hline RADAI score & Mean \pm SD & Not applicable & $2.3 \pm 1.7$ \\
\hline
\end{tabular}

Values are the mean \pm standard deviation (SD) for continuous variables and $\mathrm{N}(\%)$ for categorical variables.

Laboratory studies were collected in non-fasting conditions.

SLAQ = Systemic Lupus Activity Questionnaire; RADAI = Rheumatoid Arthritis Disease Activity Index .

* 73 RA subjects $(76.0 \%)$ had missing data on statin use. Of the 23 RA subjects with available data on statin use, 16 (69.6\%) were on statin therapy.

*** 3 SLE subjects and 3 RA subjects were missing data on current use of prednisone therapy; percentages noted excluded those with missing data.

Clin Rheumatol. Author manuscript; available in PMC 2019 February 01. 


\section{Table 2}

10-year Framingham Cardiovascular Risk Scores and 2013 ACC/AHA Cardiovascular Risk Scores in SLE and RA

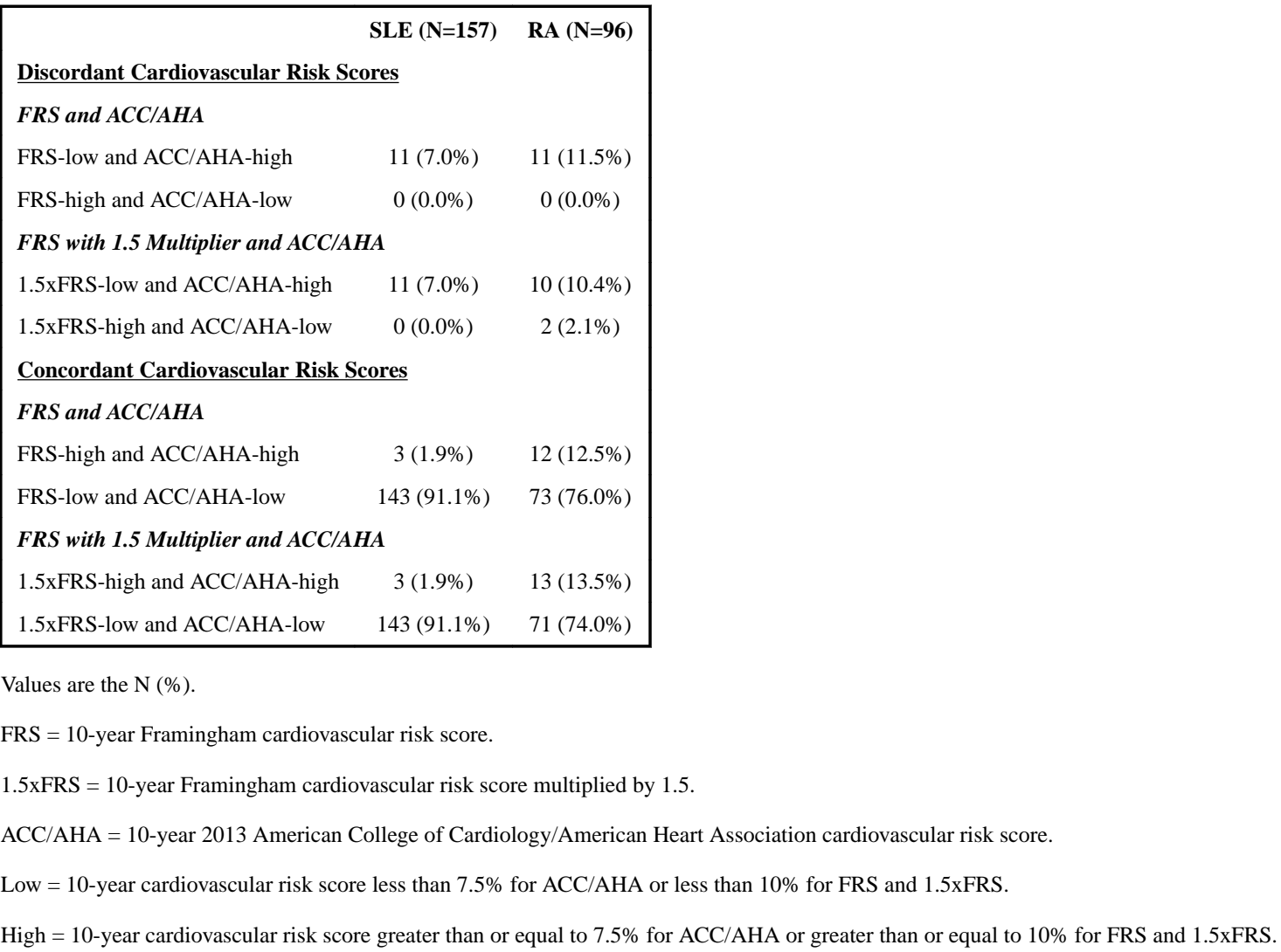


Table 3

Comparison of SLE and RA Subjects with FRS-low/ACC-high Discordant versus Concordant 10-year Framingham Cardiovascular Risk Scores and 2013 ACC/AHA Cardiovascular Risk Scores

\begin{tabular}{|c|c|c|c|c|}
\hline & & FRS-low/ACC-high Discordant (N=22) & Concordant $(\mathbf{N}=\mathbf{2 3 1})$ & p value \\
\hline \multicolumn{5}{|l|}{ Demographic Characteristics } \\
\hline Age (years) & Mean \pm SD & $65.4 \pm 7.2$ & $52.2 \pm 9.3$ & $<0.01$ \\
\hline Female & $\mathrm{N}(\%)$ & $20(90.9 \%)$ & $206(89.2 \%)$ & 0.80 \\
\hline African-American race & $\mathrm{N}(\%)$ & $5(22.7 \%)$ & $17(7.4 \%)$ & 0.02 \\
\hline \multicolumn{5}{|l|}{ Cardiovascular Clinical Characteristics } \\
\hline Body mass index $\left(\mathrm{kg} / \mathrm{m}^{2}\right)$ & Mean \pm SD & $28.9 \pm 6.0$ & $26.8 \pm 6.3$ & 0.15 \\
\hline Systolic blood pressure (mm Hg) & Mean \pm SD & $140 \pm 18$ & $121 \pm 18$ & $<0.01$ \\
\hline Diastolic blood pressure (mm Hg) & Mean \pm SD & $73 \pm 13$ & $70 \pm 13$ & 0.41 \\
\hline Total cholesterol (mg/dL) & Mean \pm SD & $179 \pm 29$ & $185 \pm 39$ & 0.51 \\
\hline LDL cholesterol (mg/dL) & Mean \pm SD & $93 \pm 23$ & $96 \pm 33$ & 0.66 \\
\hline HDL cholesterol (mg/dL) & Mean \pm SD & $61 \pm 17$ & $64 \pm 19$ & 0.50 \\
\hline Triglycerides (mg/dL) & Mean \pm SD & $126 \pm 41$ & $121 \pm 67$ & 0.69 \\
\hline Current smoker & $\mathrm{N}(\%)$ & $1(4.6 \%)$ & $14(6.1 \%)$ & 0.77 \\
\hline Diabetes & $\mathrm{N}(\%)$ & $8(36.4 \%)$ & $6(2.6 \%)$ & $<0.01$ \\
\hline Current use of anti-hypertensive medication & $\mathrm{N}(\%)$ & $15(68.2 \%)$ & $85(36.8 \%)$ & $<0.01$ \\
\hline \multicolumn{5}{|l|}{ Rheumatologic Clinical Characteristics } \\
\hline hsCRP (mg/L) & Mean \pm SD & $7.3 \pm 10.4$ & $3.6 \pm 5.9$ & 0.01 \\
\hline Current use of prednisone therapy & $\mathrm{N}(\%)$ & $6(27.3 \%)$ & $91(40.4 \%)^{*}$ & 0.23 \\
\hline $\begin{array}{l}\text { Current daily prednisone dose among } \\
\text { prednisone users (mg) }\end{array}$ & Mean \pm SD & $7.3 \pm 2.3$ & $7.5 \pm 4.6$ & 0.91 \\
\hline Disease duration (years) & Mean \pm SD & $23.5 \pm 13.1$ & $17.4 \pm 10.2$ & 0.01 \\
\hline
\end{tabular}

Values are the mean \pm standard deviation (SD) for continuous variables and $\mathrm{N}(\%)$ for categorical variables.

$\mathrm{p}$ values were calculated using chi-squared tests for categorical variables and using independent t-tests for continuous variables.

Laboratory studies were collected in non-fasting conditions.

A subject with a FRS-low/ACC-high discordant cardiovascular risk score is defined as a subject who has a 10-year Framingham cardiovascular risk score less than $10 \%$ and a 10-year 2013 ACC/AHA cardiovascular risk score greater than or equal to $7.5 \%$.

*

6 subjects with concordant cardiovascular risk scores were missing data on current use of prednisone therapy; percentage noted excluded those with missing data. 